\title{
Resummation of Infrared Divergences in the Free-Energy of Spin-two Fields
}

\section{F. T. Brandt*}

Instituto de Física, Universidade de São Paulo

E-mail: fbrandt@usp.br

\section{J. Frenkel}

Instituto de Física, Universidade de São Paulo

E-mail: jfrenkel@fma.if.usp.br

\section{G. C. McKeon}

Department of Applied Mathematics, University of Western Ontario

E-mail: dgmckeo2@uwo.ca

\section{J. B. Siqueira}

Instituto de Física, Universidade de São Paulo

E-mail: joaodif.usp.br

\begin{abstract}
We derive a closed form expression for the sum of all the infrared divergent contributions to the free-energy of a gas of gravitons. An important ingredient of our calculation is the use of a gauge fixing procedure such that the graviton propagator becomes both traceless and transverse. This has been shown to be possible, in a previous work, using a general gauge fixing procedure, in the context of the lowest order expansion of the Einstein-Hilbert action, describing non-interacting spin two fields. In order to encompass the problems involving thermal loops, such as the resummation of the free-energy, in the present work we have extended this procedure to the situations when the interactions are taken into account.
\end{abstract}

35th International Conference of High Energy Physics - ICHEP2010,

July 22-28, 2010

Paris France

${ }^{*}$ Speaker. 
The IR divergent (three or more loops) contributions to the free-energy of spin-two fields, in $d$-dimensions can be represented as [1, 2, 3, 4].

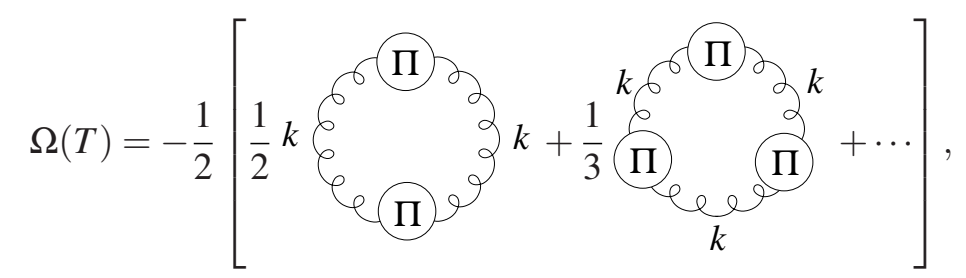

where the curly line and the blob represent the tree-level graviton propagator and the static contribution to the graviton self-energy, respectively.

A gauge invariant description of spin-two fields is provided by the Einstein-Hilbert action. Using a weak field approximation [5]

$$
g_{\mu v}=\eta_{\mu v}+\kappa h_{\mu v} ; \kappa \equiv \sqrt{32 \pi G}
$$

and introducing a gauge fixing procedure and ghosts, the tree-level graviton propagator $D_{\mu v, \alpha \beta}$ can be obtained similarly to the gauge theory of spin-one fields.

It has been shown [6] that there is a gauge fixing procedure such that the following conditions are fulfilled

$$
\begin{aligned}
\eta^{\mu v} D_{\mu v, \lambda \sigma}^{\mathrm{TT}}(k) & =0 \\
k^{\mu} D_{\mu v, \lambda \sigma}^{\mathrm{TT}}(k) & =0 .
\end{aligned}
$$

These conditions, when combined with the results for the finite temperature static graviton selfenergy [7, 8], makes the calculation of (1) much simpler that in the usual gauges.

When both the graviton propagator and the self-energy are expressed in terms of transverse and traceless tensors, each term in the integrand of Eq. (1) can be reduced to

$$
\frac{1}{\left(-|\vec{k}|^{2}\right)^{n}}\left(\frac{d(d-3)}{2}\left(\mathscr{C}^{A}\right)^{n}+(d-2)\left(\mathscr{C}^{B}\right)^{n}+\left(\mathscr{C}^{C}\right)^{n}\right)
$$

where the quantities $\mathscr{C}^{I}, I=A, B, C$ are the transverse and traceless components of the self-energy.

From Eq. (5) the sum of all the ring diagrams can be readily performed using $-\sum_{n=2}^{\infty}(-x)^{n} / n=$ $\log (1+x)-x$, so that the free-energy can be written as

$$
\begin{aligned}
\Omega(T) & =\frac{T}{2} \frac{1}{(2 \pi)^{d-1}}\left[\frac{d(d-3)}{2} I\left(\mathscr{C}^{A}\right)\right. \\
& \left.+(d-2) I\left(\mathscr{C}^{B}\right)+I\left(\mathscr{C}^{C}\right)\right]
\end{aligned}
$$

where the integral

$$
I(c) \equiv \int d^{d-1} k\left[\log \left(1+\frac{c}{|\vec{k}|^{2}}\right)-\frac{c}{|\vec{k}|^{2}}\right]
$$


can be done in a closed form (the same kind of expression also arises in the context of scalar fields). This yields the following expression for the free-energy of gravitons in $d$-dimensions

$$
\begin{aligned}
\Omega(T) & =-\frac{2 \Gamma\left(\frac{5-d}{2}\right)}{(d-1)(d-3)(2 \pi)^{d-1}}\left[\frac{2^{(5-d)} \pi^{\frac{5-d}{2}} \Gamma(d+1) \zeta(d)}{\Gamma\left(\frac{d-1}{2}\right)}\right]^{\frac{d-1}{2}}\left(G T^{d-2}\right)^{\frac{d-1}{2}} T^{d} \\
& \times\left\{\frac{d(d-3)}{2}\left(\frac{d-3}{d-1}\right)^{\frac{d-1}{2}}+(d-2)\left(\frac{d-3}{d-1}\right)^{\frac{d-1}{2}}\right. \\
& \left.+\left[\left(1-\frac{(d-2) d(d+2)}{2(d-1)}\right)\left(\frac{d-3}{d-1}\right)\right]^{\frac{d-1}{2}}\right\}
\end{aligned}
$$

This result exhibits some interesting features. First, for odd space-time dimensions it is a real and singular function; for even space-time dimensions, it is a finite and non-analytic function of $G T^{d-2}$ as one would expect for a non-perturbative quantity. However, in this case it acquires an imaginary part. For instance, for $d=4$ the third term inside the curly brackets, which can be traced back to the $C$-component of the self-energy, is equal to $(-7 / 3)^{3 / 2}$. As a result, one would conclude that the gravitational $C$-mode is unstable, since the imaginary part of the free energy is connected with the decay rate of the quantum vacuum [9]. However, a detailed investigation shows that the graviton self-energy, which is proportional to $G T^{4}$, is of the same order as the solution of the Einstein equation for the curvature tensor, when the thermal energy momentum tensor is taken into account. Therefore, by consistency, one should also take into account the curvature corrections in the analysis of instabilities of gravity at finite temperature. These corrections $[7,8]$ have the effect of adding some extra contributions to the self-energy in such a way that the $C$-mode contribution to $\Omega(T)$ would change the third term of the curly bracket of Eq. (8) to $(-7 / 3+5 / 27)^{3 / 2}$, which is still imaginary. This term may be related to an imaginary value of a thermal Jeans mass $[7,8]$, which reflects the instability of the system due to the universal attractive nature of gravity.

\section{References}

[1] J. I. Kapusta, Finite Temperature Field Theory (Cambridge University Press, Cambridge, England, 1989).

[2] M. L. Bellac, Thermal Field Theory (Cambridge University Press, Cambridge, England, 1996).

[3] A. Das, Finite Temperature Field Theory (World Scientific, NY, 1997).

[4] F. T. Brandt, J. Frenkel, D. G. C. McKeon and J. B. Siqueira, Phys. Rev. D 80, 025024 (2009).

[5] G. 't Hooft, (2002), prepared for International School of Subnuclear Physics: 40th Course: From Quarks and Gluons to Quantum Gravity, Erice, Sicily, Italy, 29 Aug - 7 Sep 2002 (this paper can be downloaded from http://www.phys.uu.nl/ thooft/lectures/erice02.pdf).

[6] F. T. Brandt, J. Frenkel, and D. G. C. McKeon, Phys. Rev. D76, 105029 (2007).

[7] A. Rebhan, Nucl. Phys. B351, 706 (1991).

[8] F. T. Brandt and J. Frenkel, Phys. Rev. D58, 085012 (1998).

[9] I. Affleck, Phys. Rev. Lett. 46, 388 (1981). 